\title{
Fucoidan promotes osteoblast differentiation via JNK- and ERK-dependent BMP2-Smad 1/5/8 signaling in human mesenchymal stem cells
}

\author{
Beom Su Kim ${ }^{1,2}$, Hyo-Jin Kang ${ }^{1}$, Ji-Yun Park ${ }^{2}$ and Jun Lee ${ }^{1,2}$
}

Fucoidan has attracted attention as a potential drug because of its biological activities, which include osteogenesis. However, the molecular mechanisms involved in the osteogenic activity of fucoidan in human alveolar bone marrow-derived mesenchymal stem cells (hABM-MSCs) remain largely unknown. We investigated the action of fucoidan on osteoblast differentiation in hABMMSCs and its impact on signaling pathways. Its effect on proliferation was determined using the crystal violet staining assay. Osteoblast differentiation was evaluated based on alkaline phosphatase (ALP) activity and the mRNA expression of multiple osteoblast markers. Calcium accumulation was determined by Alizarin red S staining. We found that fucoidan induced hABMMSC proliferation. It also significantly increased ALP activity, calcium accumulation and the expression of osteoblast-specific genes, such as ALP, runt-related transcription factor 2, type I collagen- $\alpha 1$ and osteocalcin. Moreover, fucoidan induced the expression of bone morphogenetic protein 2 (BMP2) and stimulated the activation of extracellular signal-related kinase (ERK), c-Jun N-terminal kinase (JNK) and p38 mitogen-activated protein kinase by increasing phosphorylation. However, the effect of fucoidan on osteogenic differentiation was inhibited by specific inhibitors of ERK (PD98059) and JNK (SP600125) but not p38 (SB203580). Fucoidan enhanced BMP2 expression and Smad 1/5/8, ERK and JNK phosphorylation. Moreover, the effect of fucoidan on osteoblast differentiation was diminished by BMP2 knockdown. These results indicate that fucoidan induces osteoblast differentiation through BMP2-Smad 1/5/8 signaling by activating ERK and JNK, elucidating the molecular basis of the osteogenic effects of fucoidan in hABM-MSCs.

Experimental \& Molecular Medicine (2015) 47, e128; doi:10.1038/emm.2014.95; published online 9 January 2015

\section{INTRODUCTION}

Bone development involves a balance between resorption and formation, and it is performed by osteoclasts and osteoblasts, respectively. ${ }^{1}$ Osteoporosis is one of the most common bone diseases characterized by a systemic reduction in bone mass. ${ }^{2}$ Current osteoporosis treatment strategies depend on the use of anti-resorptive and bone-forming drugs. Unfortunately, the long-term use of anti-osteoporotic medications is associated with serious side effects. ${ }^{3}$ Therefore, effective treatments that are devoid of side effects and focus on osteoblast activation are urgently needed.

Osteoblasts arise from a mesenchymal stem cell (MSC) precursor, and their differentiation is regulated by a number of growth factors, cytokines, and environmental factors. ${ }^{4,5}$ As a result, numerous researchers in the field of bone biology have studied the osteogenic-enhancing effects of compounds derived from natural products. ${ }^{6,7}$ Recently, several studies have reported that sulfated polysaccharides influence osteoblast differentiation. ${ }^{8}$
Fucoidan is a polysaccharide containing substantial proportions of L-fucose and sulfate ester groups that is mainly derived from brown algae and seaweed. ${ }^{9,10}$ For the past decade, fucoidan has been extensively studied because of its numerous biological activities, which include anti-coagulant, ${ }^{11}$ antiinflammatory ${ }^{12}$ and anti-cancer ${ }^{13}$ properties. In addition, it has been reported to induce osteogenic differentiation in human adipose-derived stem cells ${ }^{14}$ and MG-63 cells. ${ }^{15}$ However, the mechanism by which fucoidan induces this process is poorly understood.

Because there are no reports on the molecular mechanism of fucoidan in osteoblast differentiation, in this study, we aimed to determine whether it affects osteogenic differentiation via mitogen-activated protein kinases (MAPKs), several of which are essential components of the signal transduction machinery that occupy central positions in this differentiation process. ${ }^{16}$ Several MAPKs have been identified, including extracellular signal-regulated kinases (ERKs), c-Jun N-terminal protein

\footnotetext{
${ }^{1}$ Wonkwang Bone Regeneration Research Institute, Wonkwang University, Iksan, Korea and ${ }^{2}$ Research and Development Department, Bonecell Biotech Inc., Daejeon, Korea

Correspondence: Professor J Lee, Wonkwang Bone Regeneration Research Institute, Wonkwang University, Shin-Young dong, Iksan 570-749, Korea. E-mail: omslee@wku.ac.kr
}

Received 27 June 2014; revised 7 October 2014; accepted 22 October 2014 
kinase (JNK) and p38 MAPK. These three types of MAPKs regulate key transcriptional events that mediate osteoblast differentiation. ${ }^{17}$ Following activation of MAPK signaling during this differentiation process, bone morphogenetic protein 2 (BMP2)/Smad signaling is activated.

\section{MATERIALS AND METHODS}

\section{Fucoidan}

Fucoidan (a broad-range molecular weight polysaccharide) was purchased from Haewon Biotech (Seoul, Korea). Fucoidan, which is composed of $61.5 \%$ polysaccharides and $23.5 \%$ sulfate, was extracted from the brown seaweed Laminaria japonica.

\section{Cell culture}

We used MSCs from human alveolar bone marrow (hABM-MSCs), which was isolated and characterized as described previously. ${ }^{18}$ The cells were cultured in $\alpha$-Minimum Essential Medium (Gibco, Gaithersbug, MD, USA) containing $10 \%$ fetal bovine serum (Gibco) and $1 \%$ antibiotics $\left(10000 \mathrm{U} \mathrm{ml}^{-1}\right.$ penicillin $\mathrm{G}$ and $25 \mu \mathrm{g} \mathrm{ml}^{-1}$ amphotericin B; Gibco) in a humidified $\mathrm{CO}_{2}$ incubator at $37^{\circ} \mathrm{C}$. The cells were used at passages 3-6 in this study.

\section{Cell proliferation assay}

To assess the effects of fucoidan on cell proliferation, cells were seeded in a 96-well plate at a density of $3 \times 10^{3}$ cells per well. The cells were incubated in growth medium for the indicated durations at various concentrations of fucoidan $\left(0.1,0.5,1,5\right.$ and $\left.10 \mu \mathrm{g} \mathrm{ml}^{-1}\right)$. They were cultured for 1,2 and 3 days before crystal violet assays were performed. Next, the medium was removed, and the cells were washed twice with phosphate-buffered saline, fixed with $10 \%$ formaldehyde and stained with $0.5 \%(\mathrm{w} / \mathrm{v})$ crystal violet solution for $10 \mathrm{~min}$. After washing with water, the crystal violet was solubilized with $200 \mu \mathrm{l}$ of $33 \%$ acetic acid solution, and absorbance was measured at $570 \mathrm{~nm}$ using a microplate reader (SpectraMAX M3; Molecular Devices, Sunnyvale, CA, USA).

\footnotetext{
Alkaline phosphatase (ALP) staining and activity

For ALP staining, cells were seeded in a 96-well plate at a density of $5 \times 10^{3}$ cells per well and treated with various concentration of fucoidan $\left(0.1,0.5,1,5\right.$ and $\left.10 \mu \mathrm{g} \mathrm{ml}^{-1}\right)$. After 5 days of culture, the medium was removed, and the cells were washed with phosphatebuffered saline and fixed with $100 \mu \mathrm{l}$ of $10 \%$ formaldehyde at room temperature. The cells were then stained with 5-bromo-4-chloro-3indolyl-phosphate/nitro-blue tetrazolium solution (Sigma-Aldrich Co., St Louis, MO, USA) for $30 \mathrm{~min}$ at $37^{\circ} \mathrm{C}$ and imaged using a microscope (DM IL LED Fluo; Leica Microsystems, Wetzlar, Germany)

To measure ALP activity and confirm the ALP staining assay results, cells were seeded in a 96-well plate at a density of $5 \times 10^{3}$ cells per well and treated with fucoidan. After 5 days, the ALP activity assay was performed using $p$-nitrophenyl phosphate (Sigma-Aldrich Co.) as a substrate as described previously. ${ }^{19}$ Protein concentrations were measured using a bicinchoninic acid protein assay kit (Sigma-Aldrich Co.). The quantity of p-nitrophenyl phosphate was normalized to the total protein content. ALP activity was recorded in terms of $\mu \mathrm{m} \mathrm{m}^{-1}$ protein.
}

\section{Calcium accumulation assay}

Calcium accumulation was evaluated by alizarin red-sulfate (AR-S; Sigma-Aldrich Co.) staining. Cells were seeded in a 96-well plate at a density of $5 \times 10^{3}$ cells per well and cultured with continuous exposure to fucoidan for 20 days. After 20 days of fucoidan exposure, the cells were fixed with $70 \%(\mathrm{v} / \mathrm{v})$ ice-cold ethanol for $1 \mathrm{~h}$ at $4{ }^{\circ} \mathrm{C}$, stained with $40 \mathrm{~mm}$ AR-S solution, and imaged using a microscope (DM IL LED Fluo). The cells were solubilized using $10 \%(\mathrm{w} / \mathrm{v})$ cetylpyridinium chloride in $10 \mathrm{~mm}$ sodium phosphate ( $\mathrm{pH} 7.0$ ), and absorbance was measured at $562 \mathrm{~nm}$.

\section{Quantitative real-time PCR analysis}

To evaluate the effects of fucoidan on osteoblast differentiation, mRNA expression levels of several osteoblast marker genes were determined by quantitative real-time PCR using TaqMan Universal PCR Master Mix and TaqMan primers and probe sets specifically targeting runt-related transcription factor 2 (Runx2, Hs00231692 $\mathrm{m} 1)$, collagen type I $\alpha 1$ (Coll $\left.\alpha 1, \mathrm{Hs} 00164004 \_\mathrm{ml}\right)$, osteocalcin (OC, Hs01587814_g1), ALP (ALP, Hs01029144_m1), BMP2 (Hs01055564_ m1) and 18S ribosomal rRNA (Hs99999901_s1; Applied Biosystems, Carlsbad, CA, USA). All TaqMan PCR assays were performed using a StepOne Plus Real-Time PCR System (Applied Biosystems). 18S rRNA was co-amplified as an internal standard. Relative mRNA expression levels were normalized to those of the non-target shRNA sample.

\section{Western blot}

Cells were seeded at a density of $1 \times 10^{5}$ cells per 6 -cm cell culture dish and grown in maintenance medium until confluence. They were then treated with fucoidan in the presence or absence of MAPK inhibitors. Cell cultures were harvested at the indicated times and extracted at $4{ }^{\circ} \mathrm{C}$ in PRO-PREP solution (Intron Biotechnology, Inc., Sungnam, Korea). Extracts were cleared by centrifugation, and protein concentrations were determined using a protein assay kit (Bio-Rad, Hercules, CA, USA). For western blots, $10 \mu \mathrm{g}$ of each protein sample was separated by $10 \%$ SDS-polyacrylamide gel electrophoresis and electrotransferred onto nitrocellulose membranes. The blots were blocked with a buffer containing $0.05 \%$ Tween- 20 and $3 \%$ bovine serum albumin and reacted sequentially with primary and secondary antibodies. Primary antibodies against phosphorylated JNK, BMP2 (Cell Signaling Technology, Beverly, MA, USA), ERK (GeneTex, Inc., Irvine, CA, USA), p38 MAP kinase and Smad 1/5/8 (Santa Cruz Biotechnology, Santa Cruz, CA, USA) were diluted 1:1000. Horseradish peroxidase-conjugated secondary antibodies were diluted 1:7000. Finally, the membranes were developed with enhanced chemiluminescence solution and exposed to X-ray film.

\section{Knockdown of BMP2 using short hairpin RNA}

To knockdown BMP2, human BMP2 shRNA lentiviral particle (Santa Cruz Biotechnology) transduction was performed. Control transduction with non-target shRNA was also performed. Transduction of BMP2 shRNA and non-target shRNA (control shRNA) was conducted following the manufacturer's protocol. To confirm the efficiency of shRNA-mediated knockdown, BMP2 protein levels were evaluated by western blotting.

\section{Statistical analysis}

The values are expressed as the mean \pm standard deviation (s.d.), and statistical analysis was performed by one-way analysis of variance followed by the Student-Newman-Keuls multiple comparisons test for comparisons between different groups. 


\section{RESULTS}

Fucoidan promotes cell proliferation in a dose-dependent manner

To estimate the effect of fucoidan on hABM-MSCs, cell proliferation was determined. hABM-MSCs were treated with various concentrations of fucoidan $\left(0.1-10 \mu \mathrm{g} \mathrm{ml}^{-1}\right)$, and cell proliferation was analyzed using the crystal violet assay. On day 1 , hABM-MSCs incubated with fucoidan at concentrations of 5 and $10 \mu \mathrm{g} \mathrm{ml}^{-1}$ showed significantly increased proliferation in comparison with untreated (control) cells. After 2 days of culture, fucoidan induced cell proliferation in a dose-dependent manner at all tested concentrations $\left(0.1-1.0 \mu \mathrm{g} \mathrm{ml}^{-1}\right.$; Figure 1).

\section{Fucoidan promotes osteoblast differentiation}

We examined the effect of fucoidan on osteoblast differentiation using hABM-MSCs. To assess the ability of fucoidan to promote osteoblast differentiation, ALP activity was determined by staining and activity measurement. The results from the ALP staining assay demonstrated that fucoidan significantly $(P<0.01)$ induced ALP activity in the concentration range of $0.1-1.0 \mu \mathrm{g} \mathrm{ml}^{-1}$. Of the tested concentrations, the strongest effect on ALP activity was observed following the $1.0 \mu \mathrm{g} \mathrm{ml}{ }^{-1}$ fucoidan treatment. ALP activity was reduced at 5 and 10 $\mu \mathrm{g} \mathrm{ml}^{-1}$ fucoidan (Figures $2 \mathrm{a}$ and $\mathrm{b}$ ). In addition, quantitative real-time PCR revealed that mRNA expression levels of the osteoblast marker genes Runx2, Colla1, OC and ALP were significantly increased following treatment with $1 \mu \mathrm{g} \mathrm{ml}{ }^{-1}$ fucoidan (Figure 2c).

\section{Fucoidan promotes calcium accumulation}

Calcium accumulation in the extracellular matrix is a phenotypic marker of the final stages of osteoblast differentiation. Therefore, to examine whether fucoidan promotes calcium accumulation, hABM-MSCs were treated with various concentration of fucoidan $\left(0.1-10 \mu \mathrm{g} \mathrm{ml}^{-1}\right)$ and subjected to AR-S staining assays. Calcium accumulation in hABM-MSCs cultured for 15 days in the presence of fucoidan is shown in Figures $3 \mathrm{a}$ and $\mathrm{b}$. Calcium deposition was significantly increased by the fucoidan treatment at concentrations ranging from 0.1 to $10 \mu \mathrm{g} \mathrm{ml}^{-1}$. The greatest calcium deposition was observed following treatment with $5 \mu \mathrm{g} \mathrm{ml}^{-1}$ fucoidan. Calcium deposition was slightly reduced by the treatment with $10 \mu \mathrm{g} \mathrm{ml}^{-1}$ fucoidan.

Effects of fucoidan MAPK and Smad 1/5/8 phosphorylation and $B M P 2$ mRNA expression

To explore the involvement of MAPKs in fucoidan-induced osteoblast differentiation, activation of MAPKs was examined by western blotting with specific antibodies. We also explored the mRNA expression of BMP2, which is a key component of mitogenic signaling pathways. As shown in Figure 4a, fucoidan $\left(1 \mu \mathrm{g} \mathrm{m}^{-1}\right)$ treatment activated JNK, ERK and p38 within 30 min, as evidenced by increased protein phosphorylation. Furthermore, the level of phosphorylated Smad 1/5/8 was

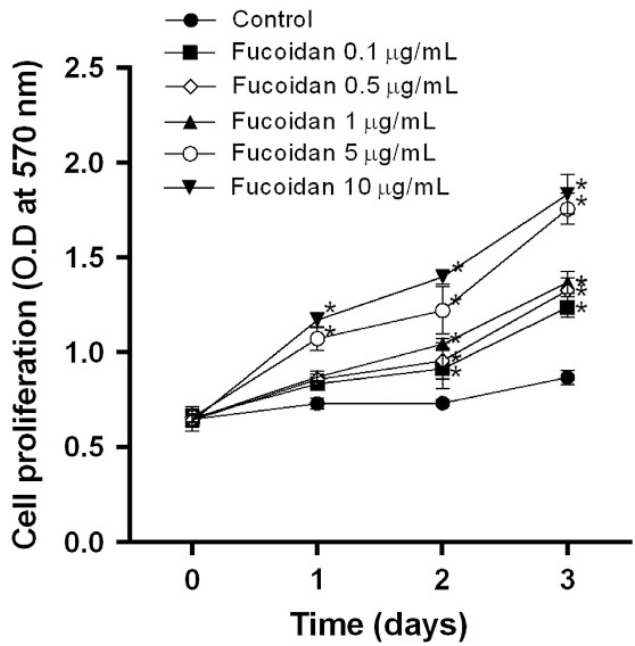

Figure 1 Effect of fucoidan on human alveolar bone marrow-derived mesenchymal stem cell proliferation. The crystal violet assay was performed on cells cultured with $0.1-10 \mu \mathrm{g} \mathrm{ml}^{-1}$ fucoidan for 1,2 and 3 days. All data represent the mean \pm s.d. of three independent experiments. ${ }^{*} P<0.05$ compared with control cells.

strongly increased after $30 \mathrm{~min}$ of treatment with fucoidan (Figure 4b). BMP2 mRNA expression was increased approximately twofold by treatment with fucoidan for 3 days (Figures $4 \mathrm{c}$ and $\mathrm{d}$ ).

\section{Effects of MAPK inhibitors on fucoidan-induced osteoblast} differentiation

To determine whether MAPKs play a role in fucoidan-induced osteoblast differentiation in hABM-MSCs, cells treated with specific inhibitors of JNK, ERK and p38 were subjected to ALP staining analysis. As shown in Figure 5, the JNK inhibitor SP600125 and ERK inhibitor PD98059 reversed the effect of fucoidan on osteoblast differentiation. However, the p38 inhibitor SB203580 had no effect on fucoidan-induced osteoblast differentiation.

MAPK inhibitors prevent mRNA expression of fucoidaninduced osteoblast marker genes

Next, we examined whether the effects of fucoidan on several osteoblast markers were mediated via MAPK signaling. The mRNA expression levels of Runx2, Col1 $\alpha 1, O C$ and ALP were evaluated in hABM-MSCs treated with specific inhibitors of JNK, ERK and p38 MAPKs. As shown in Figure 6, fucoidaninduced increases in Runx2, Col1 $\alpha 1$ and $A L P$ gene expression were attenuated by the JNK inhibitor SP600125 and ERK inhibitor PD98059. In contrast, the p38 inhibitor SB203580 did not affect fucoidan-induced gene expression. These observations strongly suggest that the stimulatory effect of fucoidan on hABM-MSC osteoblast differentiation is mediated at least in part through JNK and ERK signaling. 
a Fucoidan $(\mu \mathrm{g} / \mathrm{mL})$
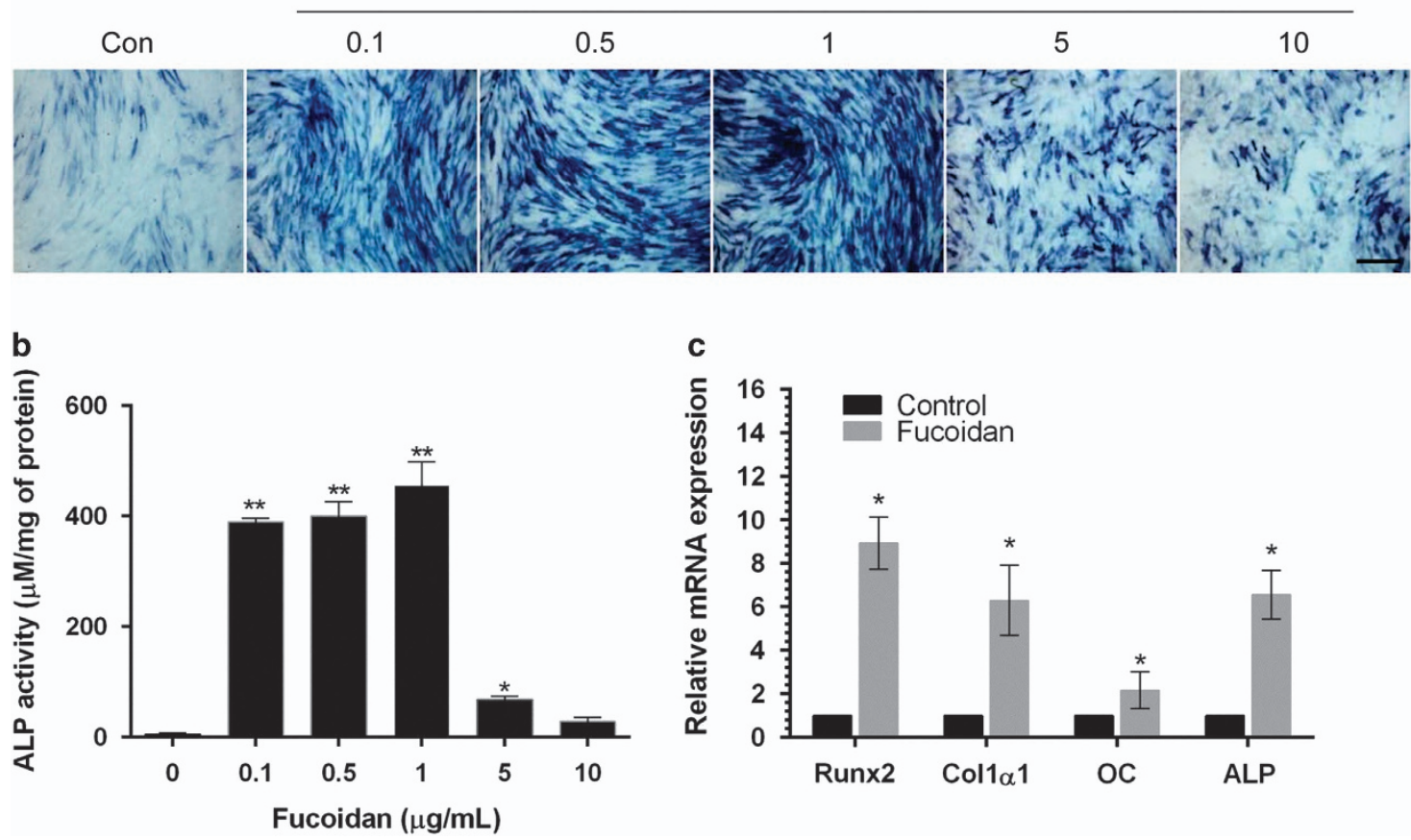

Figure 2 Effects of fucoidan on osteogenic differentiation. Confirmation of osteogenic differentiation by (a) alkaline phosphatase (ALP) staining and (b) ALP activity in human alveolar bone marrow-derived mesenchymal stem cells cultured for 5 days with fucoidan $(0.1-10 \mu \mathrm{g} \mathrm{ml}-1)$. Fucoidan at concentrations of $0.1,0.5$ and $1 \mu \mathrm{g} \mathrm{ml} \mathrm{m}^{-1}$ strongly induced ALP activity. (c) Osteoblast differentiation was confirmed by measuring mRNA expression using real-time quantitative PCR on RNA isolated from cells treated with $1 \mu \mathrm{gl} \mathrm{ml}^{-1}$ fucoidan. Scale bar $=500 \mu \mathrm{m}$. All data represent the mean \pm s.d. of three independent experiments. ${ }^{*} P<0.05$ and ${ }^{* *} P<0.01$ compared with control (Con) cells.

a

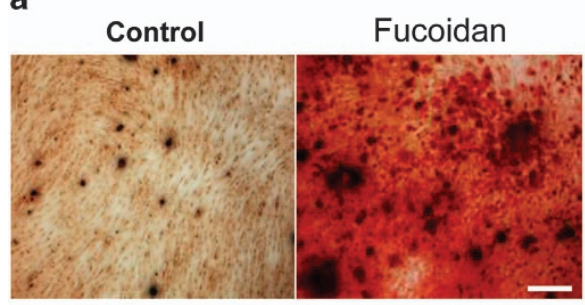

b

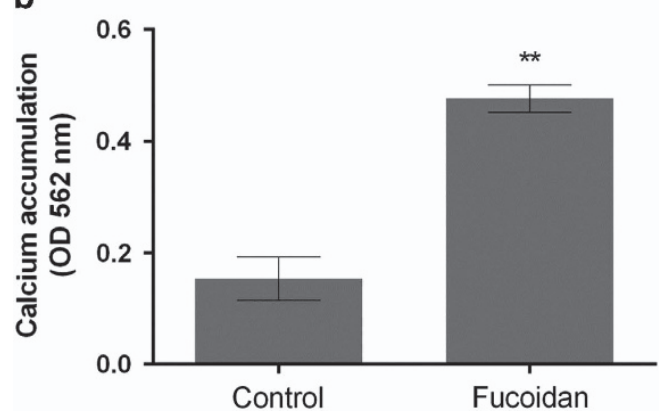

Figure 3 Effects of fucoidan on osteogenic differentiation. (a) Determination of calcium accumulation using AR-S in human alveolar bone marrow-derived mesenchymal stem cells cultured for 21 days with fucoidan $(1 \mu \mathrm{g} \mathrm{ml}-1)$. (b) Optical density measured at $562 \mathrm{~nm}$ of stained sections solubilized with cetylpyridinium chloride solution. Scale bar $=200 \mu \mathrm{m}$. All data represent the mean \pm s.d. of five independent experiments. ${ }^{* *} P<0.01$ compared with control cells.

\section{Activation of JNK and ERK occurs upstream of Smad 1/5/8 and BMP2 signaling}

In the present study, we observed that fucoidan increased Smad 1/5/8 phosphorylation (Figure $4 \mathrm{~b}$ ) and enhanced BMP2 mRNA expression (Figures $4 \mathrm{c}$ and $\mathrm{d}$ ). To evaluate whether MAPKs interact with Smad 1/5/8 or BMP2 signaling, we assessed Smad 1/5/8 phosphorylation and BMP2 mRNA expression in cells treated with specific inhibitors of JNK, ERK and p38 MAPKs. Interestingly, inhibition of the JNK-ERK signaling pathway prevented the fucoidan-induced phosphorylation of Smad 1/5/ 8 and BMP2 gene expression, whereas these effects were not observed in the presence of the p38 MAPK inhibitor (Figures $7 \mathrm{a}-\mathrm{c})$. These data clearly indicate that JNK and ERK are involved in signaling events that mediate Smad $1 / 5 / 8$ and BMP2 signaling in fucoidan-induced osteoblast differentiation in hABM-MSCs.

\section{BMP2 knockdown inhibits fucoidan-induced osteoblast differentiation}

To understand the influence of BMP2 knockdown on fucoidan-induced osteoblast differentiation in hABM-MSCs, cells were transfected with BMP2-specific shRNA lentiviral particles. The western blot data showed that BMP2 expression was reduced in the BMP2 shRNA-transfected cells by approximately $78 \%$ in comparison with the cells treated with control shRNA (Figures $8 \mathrm{a}$ and $\mathrm{b}$ ). BMP2 expression was significantly 
a
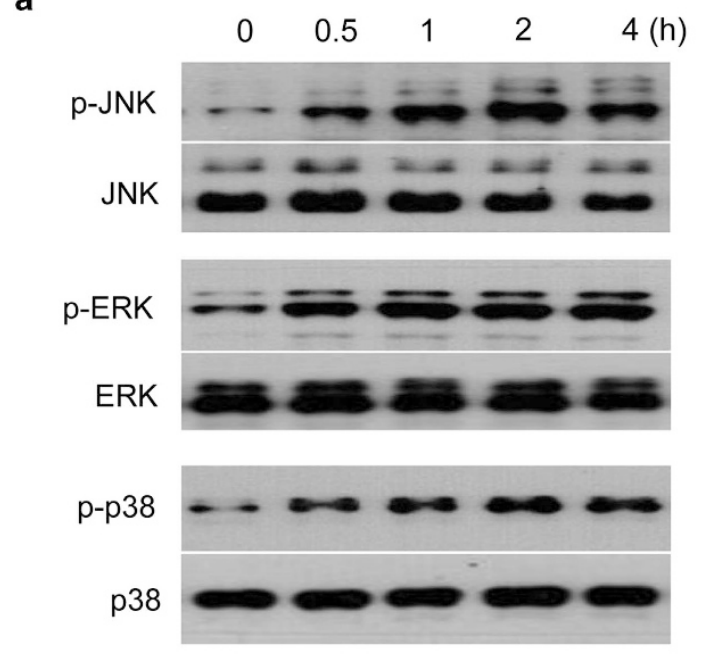

b

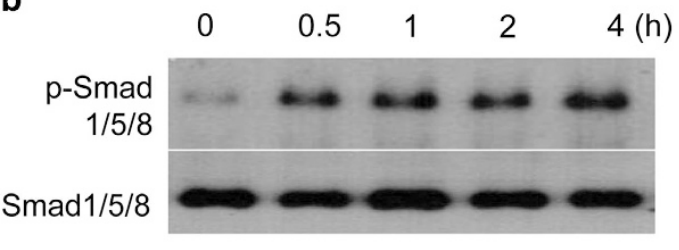

C
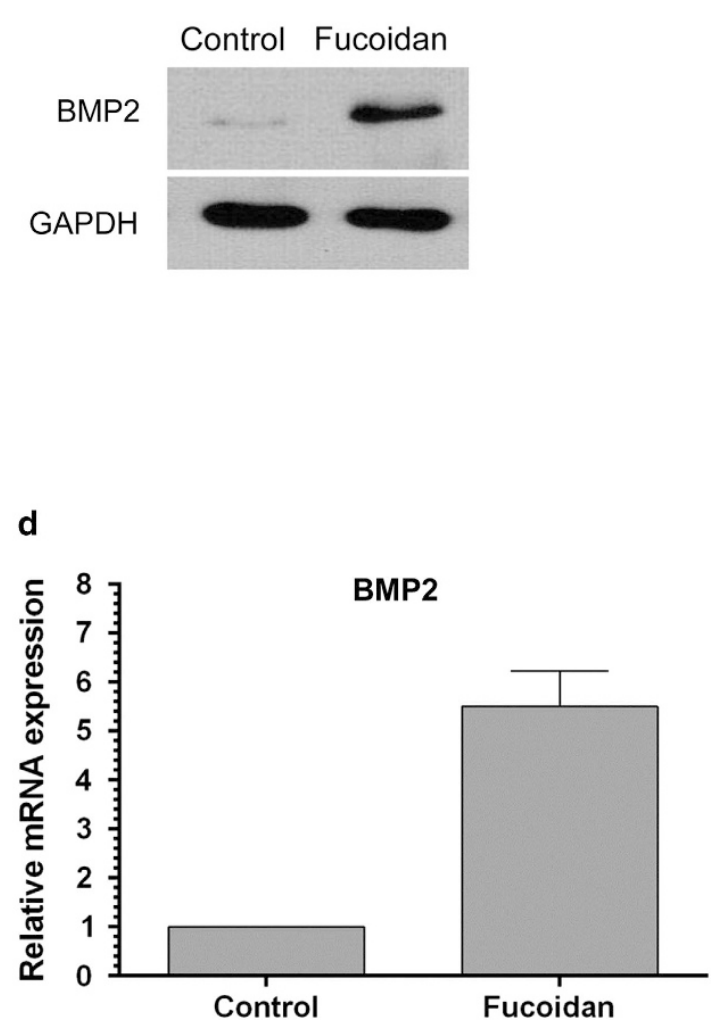

Figure 4 Effects of fucoidan on the phosphorylation of members of the mitogen-activated protein kinase family (a) and Smad 1/5/8 (b) in human alveolar bone marrow-derived mesenchymal stem cells. Cells were treated with $1 \mu \mathrm{g} \mathrm{ml}^{-1}$ fucoidan, and protein expression was measured by western blot. To evaluate the effect of fucoidan on bone morphogenetic protein 2 (BMP2) expression, cells were treated with $1 \mu \mathrm{g} \mathrm{ml}^{-1}$ fucoidan for 3 days. Protein expression was analyzed by western blot (c), and BMP2 mRNA expression was analyzed by realtime quantitative PCR (d). Fucoidan induced the phosphorylation of c-Jun N-terminal kinase (JNK), extracellular signal-related kinase (ERK) and p38. Furthermore, Smad $1 / 5 / 8$ phosphorylation was significantly increased by fucoidan treatment for 30 min. Fucoidan also increased BMP2 protein and mRNA levels after $3 \mathrm{~h}$. All data represent the mean \pm s.d. of three independent experiments. GAPDH, glyceraldehyde 3-phosphate dehydrogenase.

increased by fucoidan treatment and significantly (approximately 2.8 -fold) reduced by the BMP2-specific shRNA. The influence of BMP2 knockdown on ALP activity in hABMMSCs was determined after 5 days of cultivation in the presence or absence of fucoidan. ALP expression in BMP2specific shRNA-transfected cells was significantly reduced in comparison with that of control shRNA-transfected cells. Furthermore, pre-treatment of hABM-MSCs with the JNK inhibitor SP600125 or ERK inhibitor PD98059 led to a synergistic reduction in ALP expression following the administration of BMP2-specific shRNA (Figure 8c).

\section{DISCUSSION}

Fucoidan is a vegetal sulfated polysaccharide extracted from brown seaweed that has attracted significant research attention because of its extensive bioactivity. ${ }^{11-13}$ Recently, a small number of studies have reported the effects of fucoidan osteoblast differentiation in human osteoblast, ${ }^{6}$ osteoblast-like $(\mathrm{MG}-63)^{15}$ and adipose-derived stem cells. ${ }^{14}$ However, the effects of fucoidan on osteoblast differentiation in hABM-MSCs and the detailed signaling mechanisms involved in these effects are not well understood. In this study, we focused on the effects of fucoidan on osteoblast differentiation and associated bone formation signaling via MAPKs, Smads and BMP2. We found that fucoidan promoted proliferation and osteoblast differentiation via JNK and ERK signaling in hABM-MSCs. Our data also revealed that fucoidan stimulated osteoblast differentiation through Smad $1 / 5 / 8$ and BMP2 signaling.

A number of studies have reported conflicting effects of fucoidan on in vitro cell growth. For example, several reports have demonstrated an anti-proliferative effect of fucoidan in several tumor cell lines, including human hepatoma cells ${ }^{20}$ and lung carcinoma cells. ${ }^{21}$ In contrast, other studies have reported a proliferative effect of fucoidan in human endothelial cells. ${ }^{22}$ According to Cho et al., fucoidan does not significantly affect MG-63 cell proliferation. ${ }^{15}$ Taken together, these findings reveal that the effects of fucoidan on proliferation vary with concentration and with the tested cell line. Our findings indicated that fucoidan enhanced hABM-MSC proliferation in a dose-dependent manner, demonstrating its ability to promote proliferation in hABM-MSCs. Although we did not 


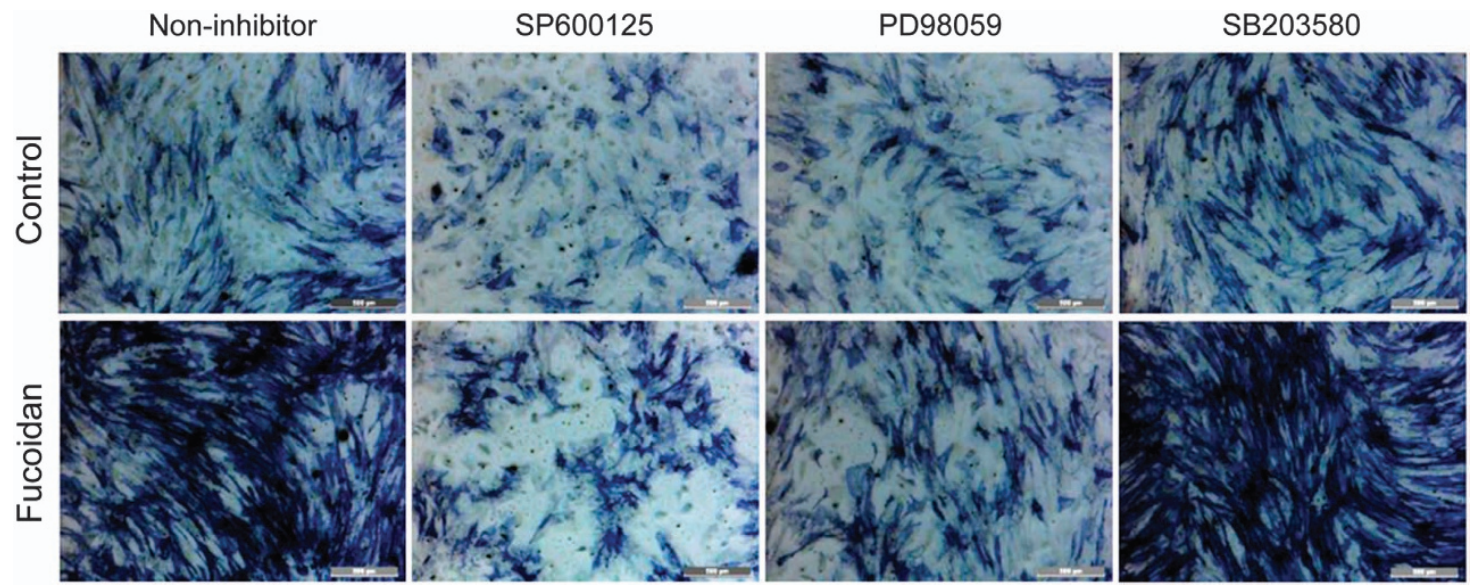

Figure 5 Evaluation of osteoblast differentiation using alkaline phosphatase staining. Human alveolar bone marrow-derived mesenchymal

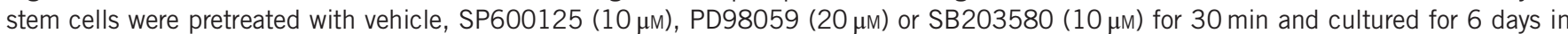
the presence of $1 \mu \mathrm{g} \mathrm{ml}^{-1}$ fucoidan. Inhibition of c-Jun $\mathrm{N}$-terminal kinase and extracellular signal-related kinase mitogen-activated protein kinases prevented fucoidan-induced osteoblast differentiation. Scale bar $=200 \mu \mathrm{m}$. Representative example of three experiments.
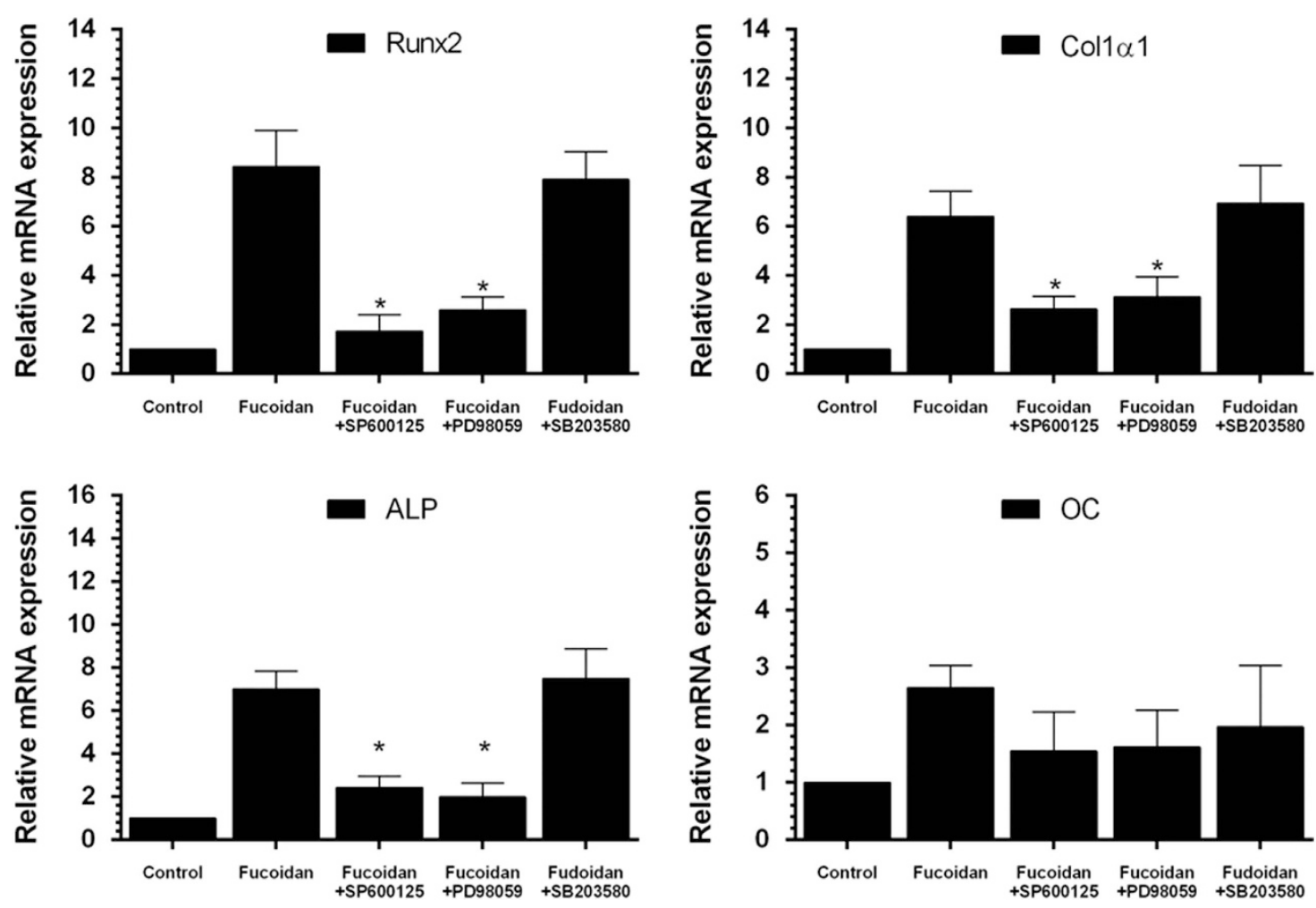

Figure 6 Expression of osteogenic differentiation marker genes after treatment with fucoidan and mitogen-activated protein kinase inhibitors. Human alveolar bone marrow-derived mesenchymal stem cells were pretreated with SP600125 (10 $\mu \mathrm{m})$, PD98059 (20 $\mu \mathrm{m})$ or SB203580 $(10 \mu \mathrm{m})$ for $30 \mathrm{~min}$ and cultured for 3 days in the presence of $1 \mu \mathrm{g} \mathrm{ml}^{-1}$ fucoidan. mRNA expression levels of several osteoblast differentiation-related genes were evaluated by quantitative real-time PCR. The expression levels of each osteoblast marker gene were normalized to that of $18 \mathrm{~S}$ rRNA, and the relative expression level was normalized to that of the control cells. SP600125 and PD98059 inhibited the fucoidan-induced mRNA expression of Runx2, Col1 1 and ALP but not OC. SB203580 did not have a significant effect on the mRNA expression of Runx2, Col1 1 , OC or $A L P$. All data represent the mean \pm s.d. of three independent experiments. * $P<0.05$ compared with control cells.

explore the exact mechanism through which fucoidan enhances proliferation, this proliferative effect may be explained by the heparin-like properties of this polysaccharide, which include growth factor binding, protection and promotion. ${ }^{23,24}$
We also determined whether fucoidan promoted osteoblast differentiation in hABM-MSCs. Changotade et al. ${ }^{24}$ have demonstrated that low-molecular-weight fucoidan has a synergistic effect on osteoblast differentiation in adipose-derived 
a

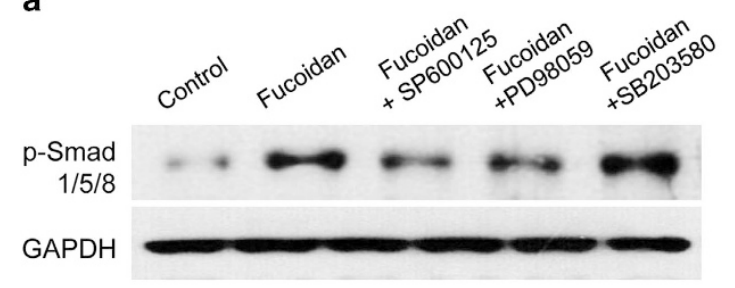

b

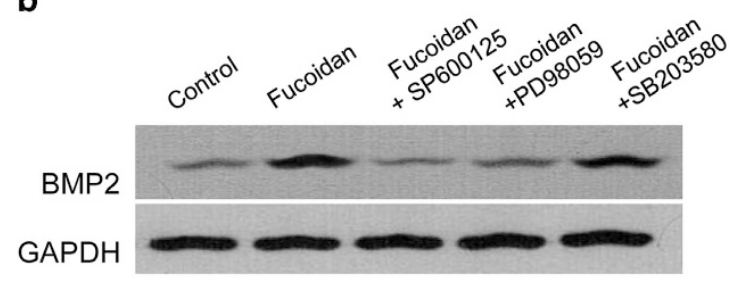

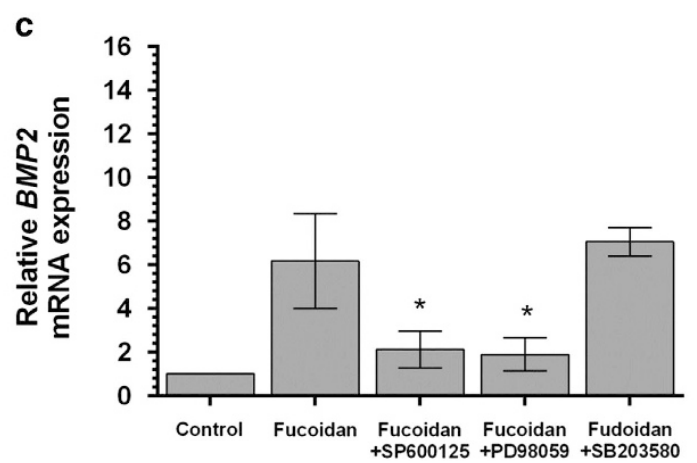

Figure 7 Effects of mitogen-activated protein kinase inhibitors on phosphorylation of Smad 1/5/8 and protein and mRNA expression levels of bone morphogenetic protein 2 (BMP2). Human alveolar bone marrow-derived mesenchymal stem cells pretreated with SP600125 $(10 \mu \mathrm{M})$, PD98059 $(20 \mu \mathrm{m})$ or SB203580 $(10 \mu \mathrm{M})$ for $30 \mathrm{~min}$ were cultured in the presence of $1 \mu \mathrm{g} / \mathrm{ml}$ fucoidan. Phosphorylated Smad $1 / 5 /$ 8 (a) and BMP2 protein expression (b) were analyzed by western blot. In addition, the mRNA expression level of BMP2 (c) was determined by real-time quantitative PCR (expression was normalized to 18S rRNA, and the relative expression level was normalized to that of the control cells). Fucoidan-induced Smad 1/5/8 phosphorylation was inhibited by SP600125 and PD98059 but not by SB203580. Furthermore, fucoidan-induced changes in the BMP2 protein and mRNA levels were inhibited by SP600125 and PD98059 but not by SB203580. All data represent the mean \pm s.d. of three independent experiments. ${ }^{*} P<0.05$ compared with control cells. GAPDH, glyceraldehyde 3-phosphate dehydrogenase.

stem cells co-treated with osteoblast reagents. Cho et al. have revealed that fucoidan promotes ALP activity and mineralization, increases $O C$ mRNA expression and increases BMP2 expression in MG-63 cells. ${ }^{4}$ In the present study, we confirmed that fucoidan treatment increased ALP activity. Although cell proliferation was increased by fucoidan in a dose-dependent manner, ALP expression and activity were decreased at high doses $\left(5\right.$ and $10 \mu \mathrm{g} \mathrm{ml}^{-1}$ ). Although this study did not evaluate the reason for the dose-dependent discrepancy between the effects of fucoidan on proliferation and differentiation, this discrepancy may have been caused by complex effects on cellular signaling mechanisms that vary with fucoidan concentration. Further studies are required to clarify the mechanisms underlying the effects of fucoidan on hABM-MSC proliferation.

In addition, we found that fucoidan markedly increased calcium accumulation and the mRNA transcription of genes involved in osteoblast differentiation, such as Runx2, Col1 $\alpha 1$, $O C$ and $A L P$. Our findings strongly suggest that fucoidan stimulates osteoblast differentiation in hABM-MSCs.

To gain further insights into the mechanisms by which fucoidan promotes osteoblast differentiation, we first evaluated MAPK signaling because the JNK-ERK-p38 MAPK pathway is known to be involved in osteogenesis. ${ }^{17,25,26}$ It has been reported that JNK and ERK are involved in osteoblast differentiation induced by fibroblast growth factor. ${ }^{27}$ In addition, p38 MAPK is required for osteoblast differentiation, osteogenic marker gene induction and ALP activity. ${ }^{28,29}$ However, there is evidence that osteoblast differentiation is stimulated by activation of ERK and JNK but not by activation of p38 MAPKs. ${ }^{30}$ In the present study, we demonstrated that the JNK and ERK MAPK signaling pathways were phosphorylated during fucoidan-induced osteoblast differentiation. Furthermore, we demonstrated that the pretreatment of cells with specific JNK and ERK inhibitors, but not with a specific p38 inhibitor, abolished fucoidan-induced osteoblast differentiation. Taken together, these data indicate that fucoidan-induced osteoblast differentiation is mediated by the JNK and ERK signaling pathways.

In addition, we examined whether MAPK inhibitors abolished the effects of fucoidan on the mRNA expression of osteogenesis-related genes (Runx2, Colla1, OC and ALP). Our results demonstrated that inhibitors of JNK and ERK, but not of p38 MAPK, led to significantly decreased expression of Runx2, Colla1 and ALP. Of particular interest, $O C$ is a late marker of osteoblast differentiation that is closely related to osteoblast maturation and matrix mineralization. ${ }^{31}$ Numerous studies have reported that $O C$ expression is regulated by JNK, ERK and/or p38 MAPK. ${ }^{32-34}$ However, we did not observe a significant alteration of $O C$ gene expression in the presence of any of the MAPK inhibitors used. Although we were unable to determine the manner by which $O C$ was stimulated by 
a

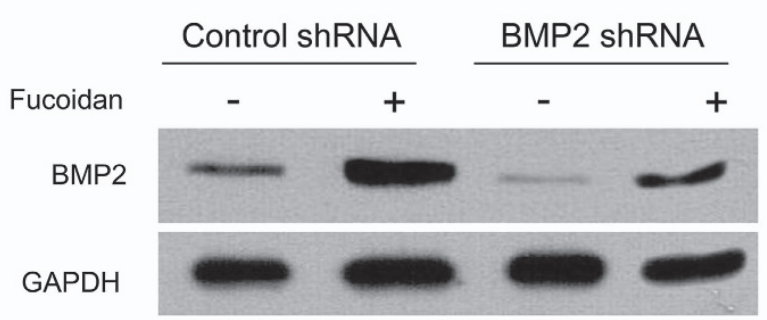

b

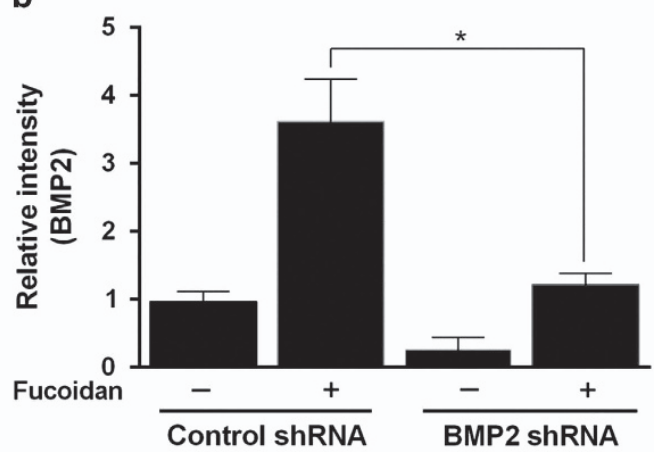

C
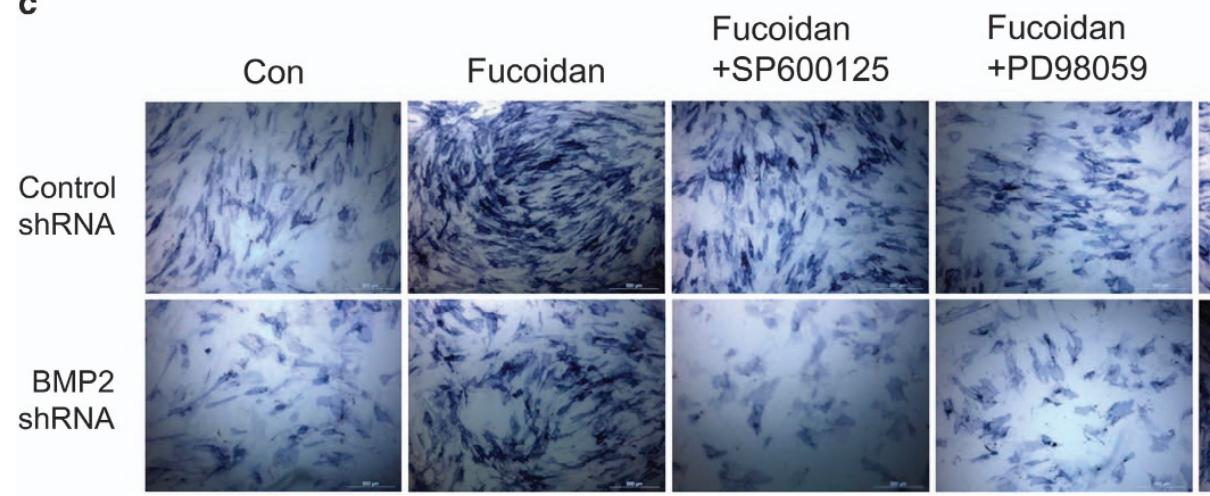

Fucoidan + SB203580

Figure 8 Fucoidan-induced osteoblast differentiation after bone morphogenetic protein 2 (BMP2) knockdown. Human alveolar bone marrow-derived mesenchymal stem cells (hABM-MSCs) were transfected with non-target short hairpin RNA (shRNA; control shRNA) and BMP2-specific shRNA (BMP2 shRNA) and cultured with $1 \mu \mathrm{g} \mathrm{ml} \mathrm{l}^{-1}$ fucoidan. BMP2 protein expression was measured by western blot (a), and relative expression was quantified using ImageJ software (http://rsb.info.nih.gov/ij/index.html) (b). In addition, cells were treated with $1 \mu \mathrm{g} \mathrm{ml}^{-1}$ of fucoidan with or without mitogen-activated protein kinase inhibitors, and alkaline phosphatase (ALP) staining was performed (c). Fucoidan-induced ALP activity was significantly decreased by BMP2 knockdown. Furthermore, in BMP2 shRNA-transfected hABMMSCs, fucoidan-induced ALP activity was significantly decreased by treatment with SP600125 (10 $\mu \mathrm{m})$ or PD98059 (20 $\mu \mathrm{m})$ but not with SB203580 (10 $\mu \mathrm{m})$. All data represent the mean \pm s.d. of three independent experiments. Scale bar $=200 \mu \mathrm{m}$. ${ }^{*} P<0.05$ compared with control cells. GAPDH, glyceraldehyde 3-phosphate dehydrogenase.

fucoidan independent of MAPKs, our observations suggest that it promotes osteoblast differentiation partially through JNKand ERK-dependent pathways involving Runx2, Coll $\alpha 1$ and ALP transcript expression, as well as through MAPKindependent pathways involving $O C$ transcript expression.

We found that fucoidan markedly increased phosphorylation of Smad 1/5/8 and BMP2 mRNA expression. BMPs are known to play an instrumental role in bone formation signaling pathways, ${ }^{35}$ and their effects are mediated through Smad signaling. ${ }^{36}$ During fucoidan-induced osteoblast differentiation, we found that Smad 1/5/8 phosphorylation was enhanced and BMP2 transcript expression was increased. Furthermore, fucoidan-induced Smad 1/5/8 phosphorylation and BMP2 mRNA expression were reduced by JNK and ERK inhibitors. These results suggest that the fucoidan-induced activation of JNKs and ERKs in osteoblasts is mediated by Smad 1/5/8 and BMP2 signaling. We found that osteoblast differentiation was significantly reduced by BMP2 knockdown. Furthermore, osteoblast differentiation was synergistically inhibited by BMP2 knockdown and the inhibition of JNK and ERK. These results indicate that BMP2 upregulation is important for fucoidan-induced osteoblast differentiation in hABM-MSCs.

In summary, our study demonstrated that fucoidan induced osteoblast differentiation in hABM-MSCs. We propose that fucoidan-induced osteoblast differentiation is mediated by Smad $1 / 5 / 8$ and BMP2 signaling via the JNK and ERK signaling pathways. Our findings suggest that fucoidan could represent a promising lead in the development of drugs for bone healing and osteoporosis treatment. In addition, further in vivo studies are necessary to identify the pharmacological effects of fucoidan in animal models.

\section{CONFLICT OF INTEREST}

The authors declare no conflict of interest.

\section{ACKNOWLEDGEMENTS}

This research was supported by the Basic Science Research Program through the National Research Foundation of Korea (NRF) funded by the Ministry of Education, Science and Technology (NRF-2012R1A1A2040526). 
1 Epker BN. Bone remodeling and balance and the development of osteoporosis. Parodontol Acad Rev 1968; 2: 125-135.

2 Ross PD, Davis JW, Vogel JM, Wasnich RD. A critical review of bone mass and the risk of fractures in osteoporosis. Calcif Tissue Int 1990; 46: 149-161.

3 Yu SF, Yang TS, Chiu WC, Hsu CY, Chou CL, Su YJ et al. Non-adherence to anti-osteoporotic medications in Taiwan: physician specialty makes a difference. J Bone Miner Metab 2013; 31: 351-359.

4 Hughes FJ, Turner W, Belibasakis G, Martuscelli G. Effects of growth factors and cytokines on osteoblast differentiation. Periodontology 2006; 41: 48-72.

5 Panetta NJ, Gupta DM, Longaker MT. Bone regeneration and repair. Curr Stem Cell Res Ther 2010; 5: 122-128.

6 Jang HO, Park YS, Lee JH, Seo JB, Koo KI, Jeong SC et al. Effect of extracts from safflower seeds on osteoblast differentiation and intracellular calcium ion concentration in MC3T3-E1 cells. Nat Prod Res 2007; 21: 787-797.

7 Kim BS, Kim YC, Zadeh H, Park YJ, Pi SH, Shin HS et al. Effects of the dichloromethane fraction of Dipsaci Radix on the osteoblastic differentiation of human alveolar bone marrow-derived mesenchymal stem cells. Biosci Biotechno Biochem 2011; 75: 13-19.

8 Manton KJ, Leong DF, Cool SM, Nurcombe V. Disruption of heparan and chondroitin sulfate signaling enhances mesenchymal stem cell-derived osteogenic differentiation via bone morphogenetic protein signaling pathways. Stem Cells 2007; 25: 2845-2854.

9 Chizhov AO, Dell A, Morris HR, Haslam SM, McDowell RA, Shashkov AS et al. A study of fucoidan from the brown seaweed Chorda filum. Carbohydr Res 1999; 320: 108-119.

10 Bilan MI, Grachev AA, Ustuzhanina NE, Shashkov AS, Nifantiev NE, Usov Al. Structure of a fucoidan from the brown seaweed Fucus evanescens C.Ag. Carbohydr Res 2002; 337: 719-730.

11 Mourao PA. Use of sulfated fucans as anticoagulant and antithrombotic agents: future perspectives. Curr Pharm Des 2004; 10: 967-981.

12 Angstwurm K, Weber JR, Segert A, Burger W, Weih M, Freyer D et al. Fucoidin, a polysaccharide inhibiting leukocyte rolling, attenuates inflammatory responses in experimental pneumococcal meningitis in rats. Neurosci Lett 1995; 191: 1-4.

13 Fukahori S, Yano H, Akiba J, Ogasawara S, Momosaki S, Sanada S et al. Fucoidan, a major component of brown seaweed, prohibits the growth of human cancer cell lines in vitro. Mol Med Rep 2008; 1: 537-542.

14 Park SJ, Lee KW, Lim DS, Lee S. The sulfated polysaccharide fucoidan stimulates osteogenic differentiation of human adipose-derived stem cells. Stem Cells Dev 2012; 21: 2204-2211.

15 Cho Y-S, Jung W-K, Kim J-A, Choi ILW, Kim S-K. Beneficial effects of fucoidan on osteoblastic MG-63 cell differentiation. Food Chem 2009; 116: 990-994.

16 Chen G, Deng C, Li YP. TGF-beta and BMP signaling in osteoblast differentiation and bone formation. Int J Biol Sci 2012; 8: 272-288.

17 Greenblatt MB, Shim JH, Glimcher LH. Mitogen-activated protein kinase pathways in osteoblasts. Annu Rev Cell Dev Bio I 2013; 29: 63-79.

18 Kim BS, Kim YC, Zadeh H, Park YJ, Pi SH, Shin HS et al. Effects of the dichloromethane fraction of Dipsaci Radix on the osteoblastic differentiation of human alveolar bone marrow-derived mesenchymal stem cells. Biosci Biotechnol Biochem 2011; 75: 13-19.

19 Kim BS, Kim JS, Chung YS, Sin YW, Ryu KH, Lee J et al. Growth and osteogenic differentiation of alveolar human bone marrow-derived mesenchymal stem cells on chitosan/hydroxyapatite composite fabric. J Biomed Mater Res A 2013; 101: 1550-1558.

20 Nagamine T, Hayakawa K, Kusakabe T, Takada H, Nakazato K, Hisanaga E et al. Inhibitory effect of fucoidan on Huh7 hepatoma cells through downregulation of CXCL12. Nutr Cancer 2009; 61: 340-347.

21 Boo HJ, Hyun JH, Kim SC, Kang JI, Kim MK, Kim SY et al. Fucoidan from Undaria pinnatifida induces apoptosis in A549 human lung carcinoma cells. Phytother Res 2011; 25: 1082-1086.
22 Giraux JL, Matou S, Bros A, Tapon-Bretaudiere J, Letourneur D, Fischer AM. Modulation of human endothelial cell proliferation and migration by fucoidan and heparin. Eur J Cell Biol 1998; 77: 352-359.

23 Jiao X, Billings PC, O'Connell MP, Kaplan FS, Shore EM, Glaser DL. Heparan sulfate proteoglycans (HSPGs) modulate BMP2 osteogenic bioactivity in C2C12 cells. The J Biol Chem 2007; 282: 1080-1086.

24 Changotade SI, Korb G, Bassil J, Barroukh B, Willig C, Colliec-Jouault S et al. Potential effects of a low-molecular-weight fucoidan extracted from brown algae on bone biomaterial osteoconductive properties. J Biom Mater Res A 2008; 87: 666-6675.

25 Hata K, Ikebe K, Wada M, Nokubi T. Osteoblast response to titanium regulates transcriptional activity of Runx2 through MAPK pathway. J Biom Mater Res A 2007; 81: 446-452.

26 Xiao G, Jiang D, Thomas P, Benson MD, Guan K, Karsenty G et al. MAPK pathways activate and phosphorylate the osteoblast-specific transcription factor, Cbfa1. J Biol Chem 2000; 275: 4453-4459.

27 Kook SH, Jeon YM, Park SS, Lee JC. Periodontal fibroblasts modulate proliferation and osteogenic differentiation of embryonic stem cells through production of fibroblast growth factors. J Periodontol 2014; 85: 654-654.

28 Kakita A, Suzuki A, Ono Y, Miura Y, Itoh M, Oiso Y. Possible involvement of p38 MAP kinase in prostaglandin E1-induced ALP activity in osteoblastlike cells. Prostaglandins Leukot Essent Fatty Acids 2004; 70: 469-474.

29 Suzuki A, Palmer G, Bonjour JP, Caverzasio J. Regulation of alkaline phosphatase activity by p38 MAP kinase in response to activation of Gi protein-coupled receptors by epinephrine in osteoblast-like cells. Endocrinology 1999; 140: 3177-3182.

30 Fu L, Tang T, Miao Y, Zhang S, Qu Z, Dai K. Stimulation of osteogenic differentiation and inhibition of adipogenic differentiation in bone marrow stromal cells by alendronate via ERK and JNK activation. Bone 2008; 43 : 40-47.

31 Hosoda K, Kanzaki S, Eguchi H, Kiyoki M, Yamaji T, Koshihara Y et al. Secretion of osteocalcin and its propeptide from human osteoblastic cells: dissociation of the secretory patterns of osteocalcin and its propeptide. J Bone Miner Res 1993; 8: 553-565.

32 Kondo A, Tokuda H, Matsushima-Nishiwaki R, Kuroyanagi G, Yamamoto N, Mizutani J et al. Rho-kinase limits BMP-4-stimulated osteocalcin synthesis in osteoblasts: regulation of the p38 MAP kinase pathway. Life Sci 2014; 96: 18-25.

33 Yasuda E, Tokuda H, Ishisaki A, Hirade K, Kanno Y, Hanai Y et al. PPARgamma ligands up-regulate basic fibroblast growth factor-induced VEGF release through amplifying SAPK/JNK activation in osteoblasts. Biochem Biophys Res Commun 2005; 328: 137-143.

34 Wang X, Harimoto K, Liu J, Guo J, Hinshaw S, Chang Z et al. Spata4 promotes osteoblast differentiation through Erk-activated Runx2 pathway. J Bone Miner Res 2011; 26: 1964-1973.

35 Phimphilai M, Zhao Z, Boules H, Roca H, Franceschi RT. BMP signaling is required for RUNX2-dependent induction of the osteoblast phenotype. $J$ Bone Miner Res 2006; 21: 637-646.

36 Ghosh-Choudhury N, Abboud SL, Nishimura R, Celeste A, Mahimainathan L, Choudhury GG. Requirement of BMP-2-induced phosphatidylinositol 3kinase and Akt serine/threonine kinase in osteoblast differentiation and Smad-dependent BMP-2 gene transcription. J Biol Chem 2002; 277: 33361-33368.

This work is licensed under a Creative Commons Attribution-NonCommercial-ShareAlike $\quad 3.0$

Unported License. The images or other third party material in this article are included in the article's Creative Commons license, unless indicated otherwise in the credit line; if the material is not included under the Creative Commons license, users will need to obtain permission from the license holder to reproduce the material. To view a copy of this license, visit http://creativecommons.org/licenses/by-nc-sa/3.0/ 